\title{
A Wideband Omnidirectional Dielectric Resonator Antenna Array
}

\author{
Taolin Liu ${ }^{1, ~ a, ~ H u ~ Y a n g ~}{ }^{1, b}$, Lei Gu ${ }^{2, c}$ and Fei Zhao,d \\ ${ }^{1}$ National University of Defense Technology, Changsha, China. \\ ${ }^{2}$ Southwest Electronics and Telecommunication Technology Research Institute, Chengdu, China. \\ azhaosenliu@163.com, byanghu90@163.com, gulei100121@sina.com, dbitzhaofei@163.com
}

Keywords: DRA array; Omnidirectional antenna; Wideband antenna; Wireless communication. Abstract. A wideband omnidirectional dielectric resonator antenna (DRA) array has been proposed. The simulated results show that the return loss of the DRA array is less than $-10 \mathrm{~dB}$ in the frequency band of $1.61 \mathrm{~dB}-2.92 \mathrm{~dB}(57.8 \%)$, which can cover the $2 \mathrm{G} / 3 \mathrm{G} / \mathrm{LTE}$ bands simultaneously. In order to reduce the deterioration of the omnidirectionality of the DRA array, a new means to arrange the feeding coaxial cable is proposed. Based on this means, a wideband DRA array with a good omnidirectionality is designed, whose gain variation ranges from $0.11 \mathrm{~dB}$ to $0.83 \mathrm{~dB}$ over the operating band of $1.61 \mathrm{GHz}-2.92 \mathrm{GHz}$. Besides, the maximum and minimum gain in the horizontal plane are $5.41 \mathrm{dBi}$ (at $2.73 \mathrm{GHz}$ ) and $3.25 \mathrm{dBi}$ (at $1.61 \mathrm{GHz}$ ), respectively. The proposed antenna in this article can be a good alternative in wireless communication systems.

\section{Introduction}

Owing to the development of modern wireless communication system, a better performance of omnidirectional antenna for $360^{\circ}$ coverage is needed recently. To cover the frequency bands of second generation (2G), third generation (3G) and long-term evolution (LTE) totally, the omnidirectional antenna must have a wide band of at least $45.5 \%(1.7-2.7 \mathrm{GHz})$. Meantime, the 2G/3G/LTE systems requires that not only the antenna has the maximum radiation direction in the azimuthal plane, but also high gain and good omnidirectionality must be obtained. The omnidirectionality is defined as the gain variation (maximum to minimum) in the azimuthal plane. The wideband of omnidirectional antenna can be achieved by rational design of monopole or dipole [1-3], but the conformation of these antennas are rather complicated. Besides, either the gain variation is above $2 \mathrm{~dB}$ [1-2] or the gain is less than $2 \mathrm{dBi}[3]$ in the horizontal plane.

Dielectric resonator antennas (DRAs) were originally proposed by Long et al. in 1983 [4]. Due to possessing many advantages, such as wideband, compact size, ease of excitation, multiple modes with respective radiation patterns and high radiation efficiency [5], DRAs have been widely applied to wireless communication systems. In order to realize an omnidirectional radiation in the horizontal plane, the $T M_{01 \delta}$ mode of a cylindrical DRA is excited. And the $T M_{01}$ mode of the feeding pin is also excited for widening the operating band. To obtain a higher gain in the azimuth plane, a DRA array that consists two elements is designed. Here, we proposed a novel means to arrange the position of the feeding coaxial cable to minimize its effect on the omnidirectionality of the DRA array.

\section{The design of the DRA element}

The configuration of the designed DRA element is shown in Fig.1. A cylindrical dielectric resonator is placed on the top of a metalic ground. To get a better omnidirectional radiation pattern in the horizontal plane, the $T M_{01 \delta}$ mode of a cylindrical dielectric resonator is excited. And the DRA is centrally fed by a coaxial probe from the bottom of the ground. The relative permittivity of the cylindrical DRA is 9. According to the closed-form formulation given in [6], the cylindrical DRA can resonate at around $2.45 \mathrm{GHz}$ when the diameter $d$ and height $h$ of the cylindrical DRA are 25mm and $50 \mathrm{~mm}$, separately. The antenna is simulated and optimized by the high frequency structure simulator (HFSS) which is based on the finite element method. The optimized parameters of the proposed cylindrical DRA element is shown in Table 1. 


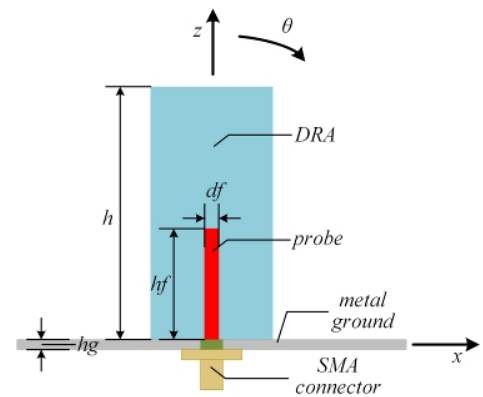

(a) Cross-sectional view

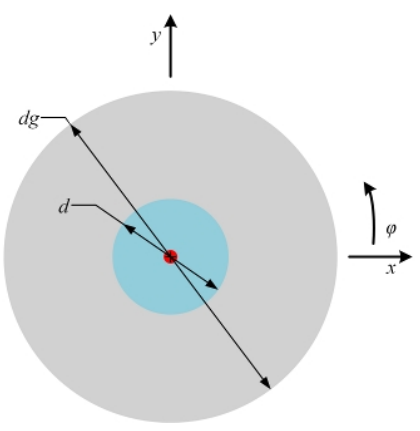

(b) Top view

Fig.1 The configuration of the designed DRA element

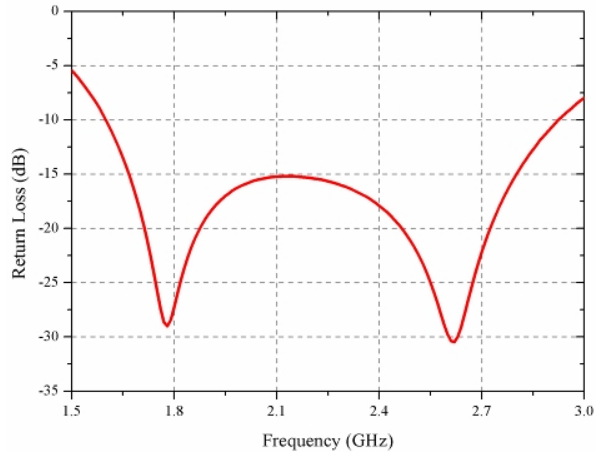

Fig.2 The simulated return loss of the cylindrical DRA

Table 1

The optimized parameters of the designed cylindrical DRA element

\begin{tabular}{|c|c|c|c|c|c|}
\hline$d(\mathrm{~mm})$ & $h(\mathrm{~mm})$ & $d f(\mathrm{~mm})$ & $h f(\mathrm{~mm})$ & $d g(\mathrm{~mm})$ & $h g(\mathrm{~mm})$ \\
\hline 24.2 & 50.1 & 2.8 & 22 & 77 & 2 \\
\hline
\end{tabular}

The simulated return loss of the optimized cylindrical DRA is shown in Fig.2, which shows that the simulated return loss is less than $-10 \mathrm{~dB}$ in the frequency band of $1.61 \mathrm{GHz}-2.92 \mathrm{GHz}(57.8 \%)$, which can totally cover the $1.7 \mathrm{GHz}-2.7 \mathrm{GHz}$ fequency band. The first resonate frequency is close to $1.78 \mathrm{GHz}$, which is caused by the feeding pin. And the second one is around $2.62 \mathrm{GHz}$, which is arose from the $T M_{01 \delta}$ mode of the DRA.

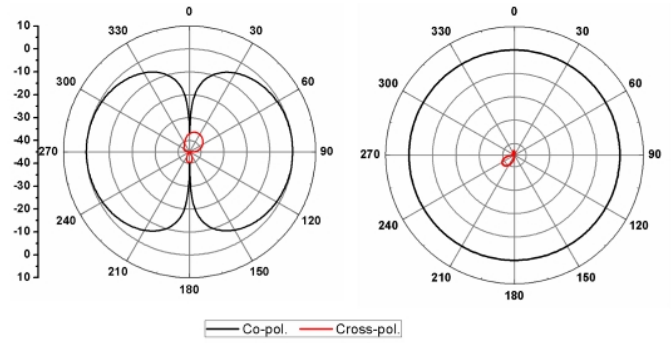

(a) E (xoz) plane (b) H (xoy) plane

Fig.3 Normalized radiation patterns at $1.61 \mathrm{GHz}$

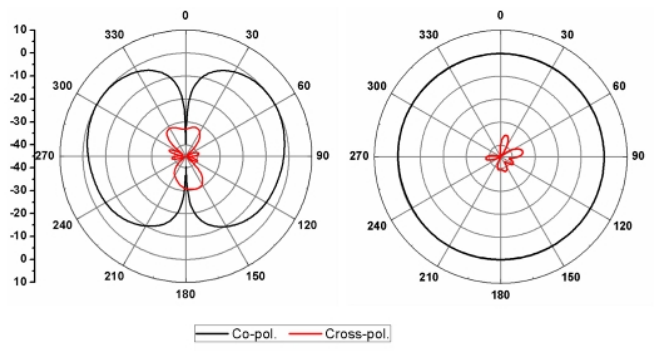

(a) E (xoz) plane $\quad$ (b) $\mathrm{H}(\mathrm{xoy})$ plane

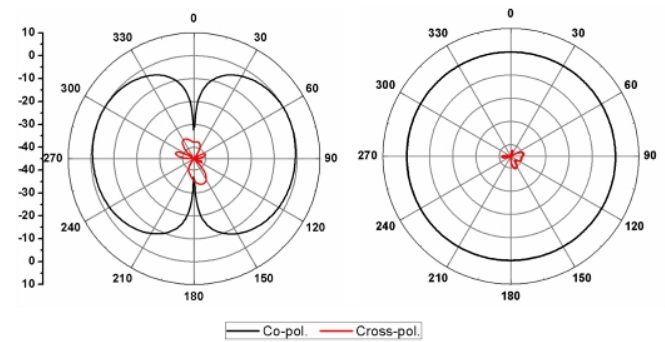

(a) E (xoz) plane

(b) $\mathrm{H}$ (xoy) plane

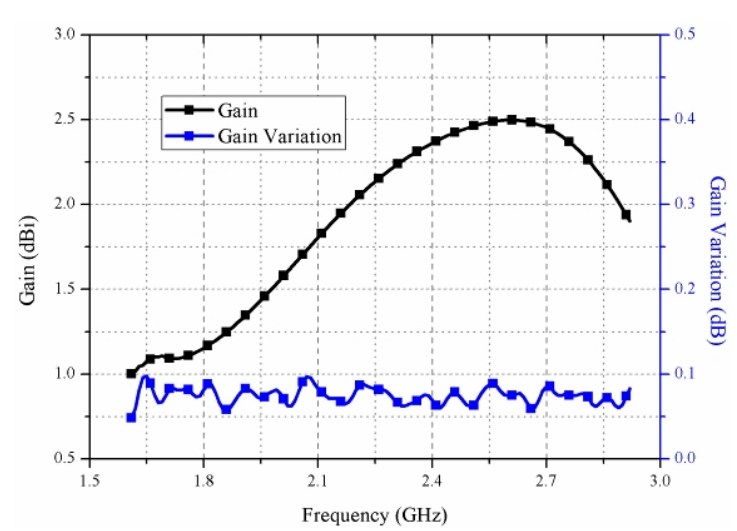

Fig.5 Normalized radiation patterns at 2.92GHz Fig.6 Simulated gain and gain variation of the DRA element in the horizontal plane

The simulated radiation patterns of the DRA element at three typical frequency points are depicted in Fig.3-Fig.5, respectively. It can be seen that the designed antenna has an omnidirectional radiation pattern in the horizontal plane over the whole operating band though the maximum radiation direction 
tilts up a little when the frequency is higher than $2.5 \mathrm{GHz}$. And the cross polarization is lower than $40 \mathrm{~dB}$ in the main radiation direction. Simulated gain and gain variation of the DRA element in the H-plane are shown in Fig.6. The gain ranges from $1.0 \mathrm{dBi}$ to $2.61 \mathrm{dBi}$ in the frequency band of $1.61 \mathrm{GHz}-2.92 \mathrm{GHz}$. And the maximum and minimum gain appear at $1.61 \mathrm{GHz}$ and $2.61 \mathrm{GHz}$, separately. The gain variation in the azimuth plane among the operating band is less than $0.1 \mathrm{~dB}$. In conclusion, a wideband cylindrical DRA element with good omnidirectionality in the horizontal plane has been designed and analyzed.

\section{The design of the DRA array}

To enhance the gain of the antenna in horizontal plane, a side-fire radiation antenna array with two identical elements that are designed in the above section has been proposed here. The ports of the two antenna elements are fed in-phase and equal-amplitude by a 1-2 power divider, which is located at the bottom of the array. According to the design principles in [6], the distance between the elements is designed and optimized, dist $=100 \mathrm{~mm}$ is chosen here. And simulated results show that the return loss of the DRA element in the array is still lower than $-10 \mathrm{~dB}$ in the frequency band of $1.61 \mathrm{GHz}-2.92 \mathrm{GHz}$. Considering the implementation in engineering, the feeding coaxial cable to the 2\# DRA element has to pass through the section between the two antenna elements, which will deteriorate the omnidirectionality of the array a lot if the coaxial cable is just arranged as Fig.7 (a) shown. When $p c=35 \mathrm{~mm}, d c=2.2 \mathrm{~mm}$, the radiation patterns at $1.61 \mathrm{GHz}, 2.5 \mathrm{GHz}$ and $2.92 \mathrm{GHz}$ are given in Fig.8Fig.10, respectively. It can be seen that the feeding coaxial cable mainly affects the horizontal radiation patterns. Compared with the condition that the coaxial cable doesn't exist, the gain variation is increased $2.4 \mathrm{~dB}$ at $2.5 \mathrm{GHz}$. Besides, the gain variation will be increased further when $d c$ is increased or $p c$ is decreased in a rational range.

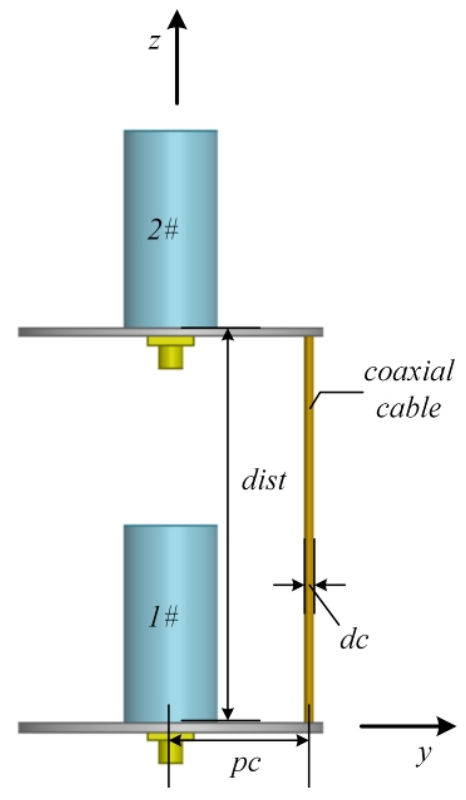

(a) The DRA array with feeding coaxial cable

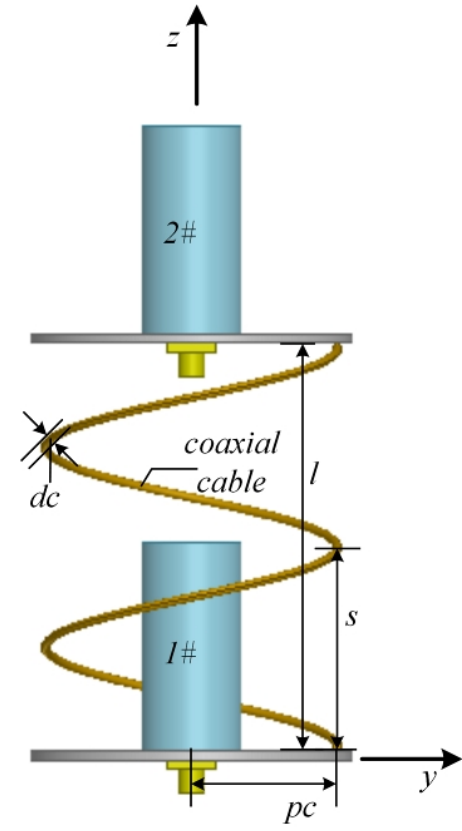

(b) The DRA array with helix-shaped feeding coaxial cable

Fig.7 The geometry of the DRA array with different feeding coaxial cable 


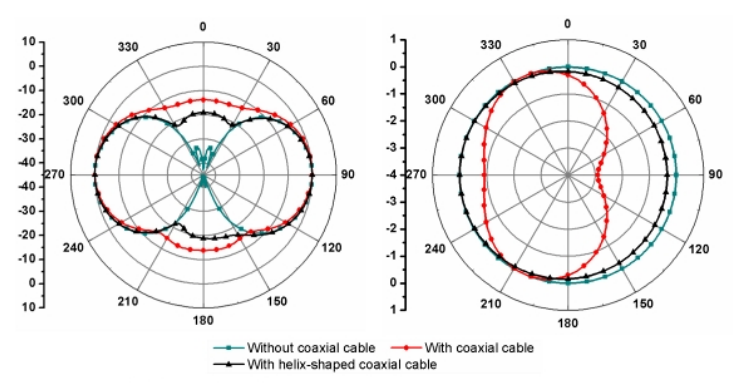

(a) E (xoz) plane

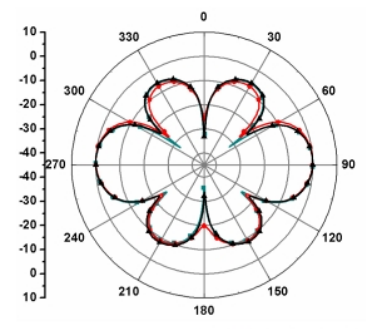

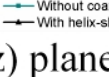

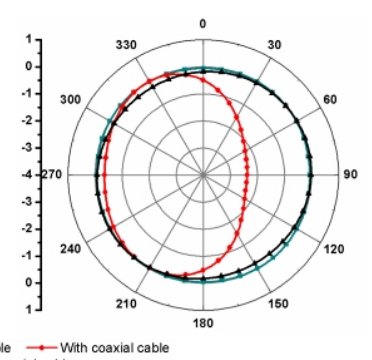

(b) $\mathrm{H}$ (xoy) plane

Fig.8 Normalized radiation patterns at $1.61 \mathrm{GHz}$

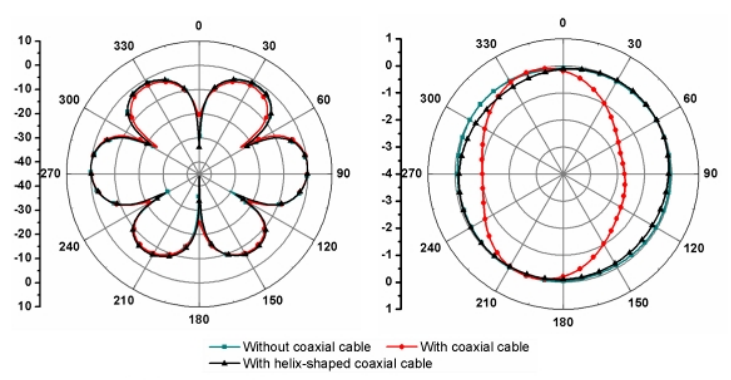

(a) E (xoz) plane

(b) $\mathrm{H}$ (xoy) plane
Fig.9 Normalized radiation patterns at $2.5 \mathrm{GHz}$

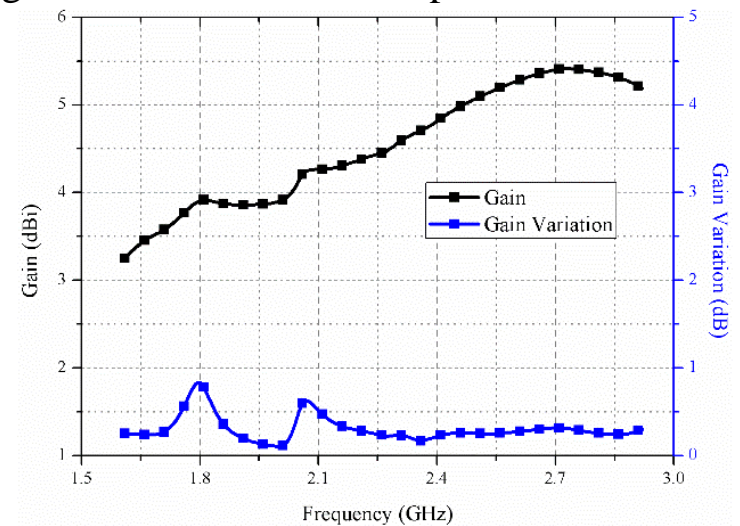

Fig.11 Simulated gain and gain variation of the DRA array with helix-shaped feeding coaxial cable in the horizontal plane

Fortunately, we get a new way to arrange the position of the feeding coaxial cable to the 2\# DRA element. Just as Fig.7 (b) shown, a helix-shaped coaxial cable centered on the $z$ axis rotates from the ground of 1\# DRA element to the ground of 2\# DRA element. The axial length of the helix $l=d i s t-h g$, the pitch $s$ and the number of turns $n$ can be determined by $s^{\times} n=l$. To avoid enlarging the size of the antenna array, the position of the feeding coaxial cable $p c$ has to satisfy $p c \leq(d g-d c) / 2$. When $p c=35 \mathrm{~mm}, d c=2.2 \mathrm{~mm}, n=2$, the omnidirectionality of the DRA array is ameliorated. To illustrate this phenomenon, the normalized radiation patterns at $1.61 \mathrm{GHz}, 2.5 \mathrm{GHz}$ and $2.92 \mathrm{GHz}$ are compared in Fig.8-Fig.10, separately. The radiation patterns of the DRA array with helix-shaped coaxial cable are more close to the ideal DRA array's. The ideal DRA array is definited as the DRA array without coaxial cable.

Fig. 11 shows that the gain variation ranges from $0.11 \mathrm{~dB}$ to $0.83 \mathrm{~dB}$ over the frequency band of $1.61 \mathrm{GHz}-2.92 \mathrm{GHz}$, and it's lower than $0.5 \mathrm{~dB}$ in the most of the operating frequency band. Besides, the maximum and minimum gain in the horizontal plane are $5.41 \mathrm{dBi}$ and $3.25 \mathrm{dBi}$, respectively.

\section{Conclusions}

Compared with the monopole/dipole antennas, DRAs have wider bandwidth and are more flexible in designing. In recent years, DRAs have been investigated further by many a researcher. While there are few papers reporting the omnidirectional DRA array. Due to its 3-D configuration, the feeding of the omnidirectional DRA array is a key problem that should be discussed. In this article, we proposed a new means to arrange the feeding coaxial cable, which can improve the omnidirectionality of the DRA array effectively in a wideband. The simulated results show that gain variation in the horizontal plane is less than $0.83 \mathrm{~dB}$ in the whole frequency band of $1.61 \mathrm{GHz}-2.92 \mathrm{GHz}$. The maximum gain of the designed DRA array can reach $5.41 \mathrm{dBi}$ in the azimuthal plane. In addition, the means that proposed here can be applied to other omnidirectional antenna arrays, too. 


\section{References}

[1] S. Abadi and N. Behdad, "An electrically small, vertically polarized ultrawideband antenna with monopole-like radiation characteristics," IEEE Antennas Wireless Propag. Lett., vol.13, pp. 742-745, Apr. 2014.

[2] M. Koohestani, J.-F. Zürcher, A. A. Moreira, and A. K. Skrivervik, "A novel, low-profile, vertically-polarized UWB antenna for WBAN,” IEEE Trans. Antennas Propag., vol. 62, no. 4, pp. 1888-1894, Apr. 2014.

[3] Ze Dong Wang, Ying Zeng Yin, Xi Yang and Jian Jun Wu, "Design of a Wideband Horizontally Polarized Omnidirectional Antenna With Mutual Coupling Method," IEEE Trans. Antennas Propag., vol. 63, no. 7, pp. 3311-3316, July. 2015.

[4] S. A. Long, M. W. McAllister, and L. C. Shen, "The resonant cylindrical dielectric cavity antenna," IEEE Trans. Antennas Propag., vol. 31, no. 3, pp. 406-412, May 1983.

[5] Taolin Liu, Hu Yang, Lei Gu, Fei Zhao and Wenlu Yin, "An omnidirectional cylindrical DRA with a cone ground for WLAN applications.", 2015 IEEE Advanced Information Technology, Electronic and Automatic Control Conference (IAEAC), 2015.

[6] Balanis C A. Antenna Theory: Analysis and Design [J]. New York, 2005, 72(7):989-990. 